\title{
Optimisation of Scanning Difference Pattern and Monopulse Feed
}

\author{
Asim Ali Khan ${ }^{1}$ and Anthony Keith Brown ${ }^{2}$ \\ ${ }^{1}$ COMSATS Institute of Information Technology, Lahore, Pakistan \\ ${ }^{2}$ University of Manchester, Manchester M60 1QD, UK \\ Correspondence should be addressed to Asim Ali Khan; drasim@ciitlahore.edu.pk
}

Received 2 February 2017; Revised 30 April 2017; Accepted 16 May 2017; Published 15 June 2017

Academic Editor: Gino Sorbello

Copyright (c) 2017 Asim Ali Khan and Anthony Keith Brown. This is an open access article distributed under the Creative Commons Attribution License, which permits unrestricted use, distribution, and reproduction in any medium, provided the original work is properly cited.

\begin{abstract}
Certain radar applications may require maintaining the difference pattern slope and twin beam shape, while main null is scanning in the presence of mutual coupling. It is also desirable in monopulse radar applications to be able to generate acceptable sum and difference patterns using single simplified feed structure. This paper focuses on these problems and provides a solution based on Intelligent $z$-Plane Boundary Condition-Particle Swarm Optimiser (IzBC-PSO) to compensate for the difference pattern degradation while scanning a small coupled array $(N=8)$. In second case, a simplified feed is proposed that only requires phase flip for $50 \%$ of elements to produce sum and difference patterns for the monopulse array, consisting of isotropic elements.
\end{abstract}

\section{Introduction}

Monopulse radar antenna is widely used in tracking and direction of arrival applications. Monopulse operation depends on the sum and difference pattern characteristics. Usually sum and difference patterns are used such that the sum pattern main beam and the difference pattern null are both pointing in the same $\theta_{o}$ direction to accurately determine the direction of arrival. For this purpose, the difference pattern slope is very important factor to identify the main null of the difference pattern in the presence of noise. Conventionally, Taylor distribution [1] is used to determine the sum pattern with the desired side lobe level (SLL) characteristics.

The conventional analytical techniques of difference pattern synthesis for linear antenna arrays include Zolotarev polynomial based approach introduced by McNamara [2] and the Bayliss distribution $[3,4]$. The Zolotarev polynomial based approach results in the optimum array radiation pattern in the Dolph-Chebyshev [5] manner. For a given number of elements and a defined Difference Pattern Beam Width (DBW) between the main null and the first null of difference pattern, Zolotarev distribution provides a radiation pattern with minimum SLL and the minimum DBW [6].
Monopulse operation requires two independent feed structures to support the desired sum and difference patterns which increase the complexity and cost of the system. It is desirable from the system design point of view to obtain a taper that simplifies the array feed. Ideally the same amplitudes across the array for both sum and difference patterns with $180^{\circ}$ phase shift across the half of the aperture.

Several approaches have been reported to achieve this goal. Hosseini and Atlasbaf [7] have reported solutions based on phase only, amplitude only, and separation only methods for medium sized array. The methods based on amplitude only and phase only gave low SLLs but required a complex feed structure. The separation only method resulted in simple feed structure but SLLs were comparatively high. An approach based on the subarrayed linear arrays is used to find the optimum sum and difference patterns [8]. The work is focused on minimizing the SLL while maximizing the slope across broadside direction.

Another approach proposed by D'Urso and Isernia [9] based on hybrid optimisation technique deals with the problem of simplifying the feeding network for optimal sum and difference patterns. The proposed technique starts with a set of sum pattern excitations. To determine the difference pattern excitations subarray clustering is performed. 
As a result of this approach difference elements have chosen for sum and difference patterns among the subarrays. The drawback of this technique is that additional elements are required in the subarrays as not all of them are used in one particular scenario (for sum or difference pattern).

To simplify the feed structure, it has been proposed to reduce the difference between the Taylor and Bayliss taper using simulation annealing optimiser [10]. The authors were partially successful as up to $50 \%$ of a common aperture distribution could be shared between the Taylor and Bayliss distribution. This then uses a common feed structure over half the array but still requires different weights for sum and difference patterns over the remaining half of an aperture.

The objective in difference pattern synthesis is to determine a pattern that has a target side lobe level (SLL) constraint and maximum difference slope. Various optimisation algorithms have been introduced to solve this problem [1115]. In these cases, medium sized arrays of isotropic radiators are considered and no pattern scanning is applied. When the main null is steered radiation pattern is degraded and target pattern features are compromised.

Several methodologies based on subarray technique have been reported to have simultaneous sum and difference patterns in a monopulse array system. An approach based on excitation matching and subarraying to produce simultaneous sum and difference patterns is presented by McNamara [15]. It was observed that the excitation matching method did not provide full control over the radiation pattern. An improvement to the excitation matching technique is introduced by Rocca et al. [16] based on the ant colony algorithm for monopulse operation. Other subarray techniques based on hybrid and evolutionary optimisation solutions have been reported in literature [17-27].

The techniques mentioned above consider either arrays with $N>10$ or continuous aperture. The work presented in this paper is divided into two cases. In Case I Intelligent $z$-Plane Boundary Condition-Particle Swarm Optimiser (IzBC-PSO) [28] is used to achieve a scanned difference pattern in the presence of mutual coupling. For this an antenna array consisting of Bowtie Dielectric Resonator Antenna (BDRA) [29] is modelled in Computer Simulation Technology-Microwave Studio (CST-MWS). In Case II a simplified feed structure is proposed using IzBC-PSO for the monopulse radar system for an isotropic array of $N=8$.

\section{Problem Setup}

For a periodic linear antenna array consisting of $N$, where $N$ is the number of elements, elements placed along $x$-axis and the Array Factor (AF) in $z$-plane are given as Schelkunoff's polynomial [6]:

$$
\operatorname{AF}(z)=\sum_{n=0}^{N-1} w_{n} z^{n}
$$

where $w_{n}$ is the complex weight coefficient, $z=e^{-j k d \sin \theta}$, $k$ is the wave number given by $2 \pi / \lambda, d$ is the interelement spacing, and $\theta$ is the pattern angle.
If a progressive phase shift is applied to scan the main beam at $\theta_{o}$ then the array element excitation of the $n$th element is given as $w_{n}=a_{n} e^{-j n \beta}$, where $a_{n}$ is the amplitude of the excitation and $\beta=j k d \sin \theta_{o}$ is the phase variation required for progressive phase shift.

$$
\begin{aligned}
& \operatorname{AF}(z)=\sum_{n=0}^{N-1} a_{n} e^{-j n \beta} z^{n} \\
& \operatorname{AF}(\theta)=\sum_{n=0}^{N-1} a_{n} e^{-j n(k d \sin \theta+\beta)}
\end{aligned}
$$

In large antenna arrays mutual coupling mostly affects the element performance across the array uniformly. To model mutual coupling effect in large antenna arrays, immerse element pattern is considered sufficient [30]. On the contrary, in small antenna arrays (i.e., $N<10$ ) the effect of mutual coupling on array elements is nonuniform and therefore mutual coupling model is complicated. There are various mutual coupling models available in literature. For this work, active element pattern was selected for its ability to model mutual coupling effects on the array radiation patterns [31, 32]. Computer Simulation Technology Microwave Studio ${ }^{\circledR}$ (CST-MWS) was used to simulate and find active element pattern for each element in the antenna array, shown in Figures 1(c)-1(d).

For an array with identical nonisotropic elements, each with radiation pattern $E(\theta)$, the total radiation pattern (TRP) is given by

$$
\operatorname{TRP}(\theta)=E(\theta) \cdot A F(\theta)
$$

The TRP in case of nonidentical active element patterns [11] is then given by

$$
\operatorname{TRP}(\theta)=\sum_{n=0}^{N-1} w_{n} E_{n}(\theta),
$$

where $E_{n}$ is the element pattern of the $n$th element. For pattern synthesis the root positions in the $z$-plane are optimised to produce a radiation pattern meeting the requirements as closely as possible [28].

2.1. Case I. A linear array of eight BDRA elements is considered for this study and details are given in Figure 1. The effective element separation is $d=0.41 \lambda$ at $4.5 \mathrm{GHz}$. For isotropic radiation pattern $w_{n}$ in (1) is replaced by Bayliss taper for $-30 \mathrm{~dB}$ SLL and $\bar{n}=3$. Figure 2 shows both patterns for $d=0.41 \lambda$ with main null scanned to broadside and $30^{\circ}$ off the broadside achieved by applying the linear phase gradient.

The optimisation goal is to achieve difference pattern with deep main null and improved difference pattern slope while minimizing the difference between the two difference peaks for a $30^{\circ}$ scanned BDRA array in the presence of mutual coupling. This scenario is studied with and without SLL restriction.

The difference peaks of the scanned pattern are indicated in Figure 3. The difference pattern slope, $\kappa(\mathrm{dB} / \mathrm{deg})$, is defined 


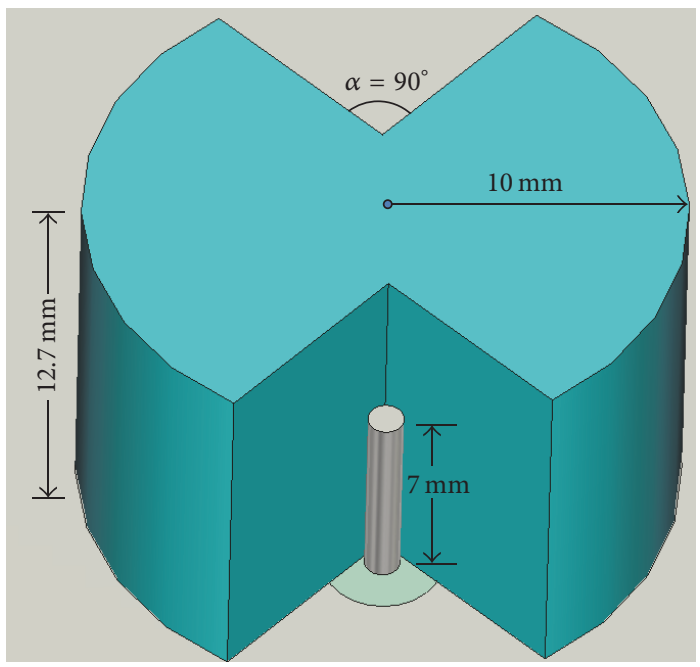

(a)

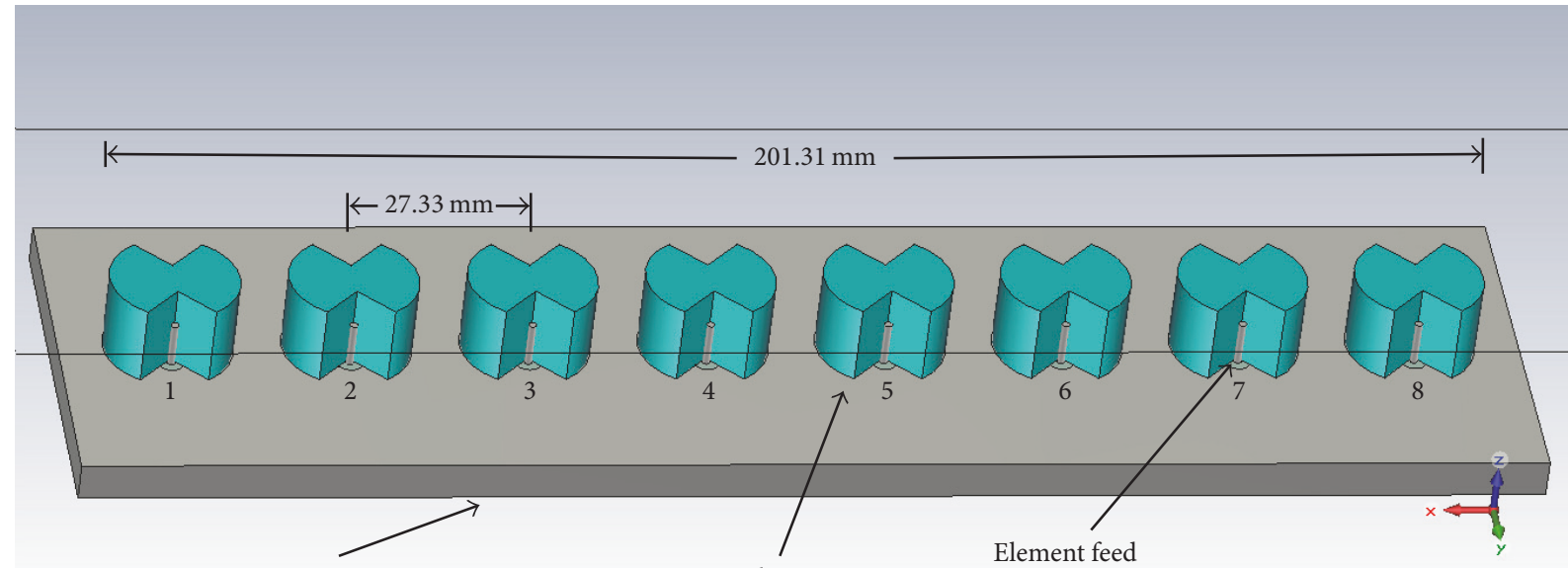

Ground plane

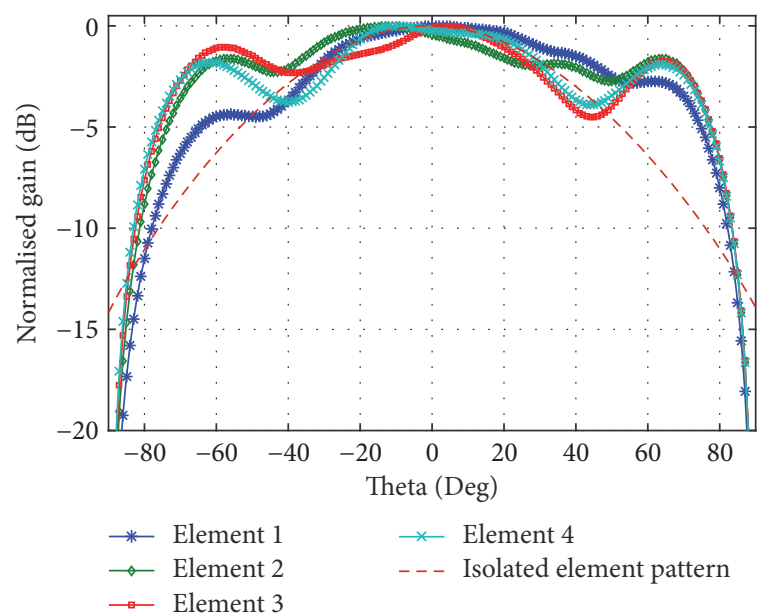

(c)

(b)

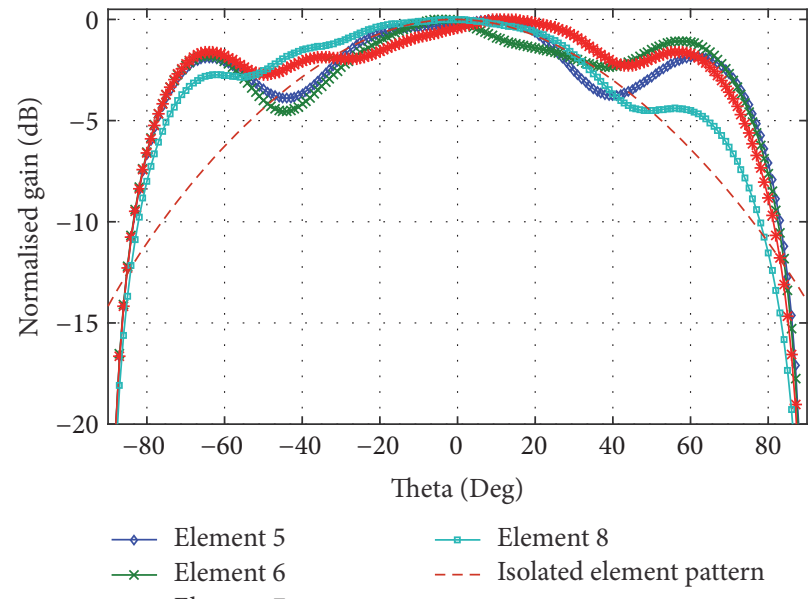

(d)

FIGURE 1: (a) Single BDRA element as presented in [29] given with dimensions, placed on $60 \times 60 \mathrm{~mm}$ ground plane, (b) BDRA array designed and simulated in CST-MWS with $d=0.41 \lambda$, and (c) simulated element patterns for elements $1-4$ and (d) for elements 5-8 along with the isolated element pattern. 


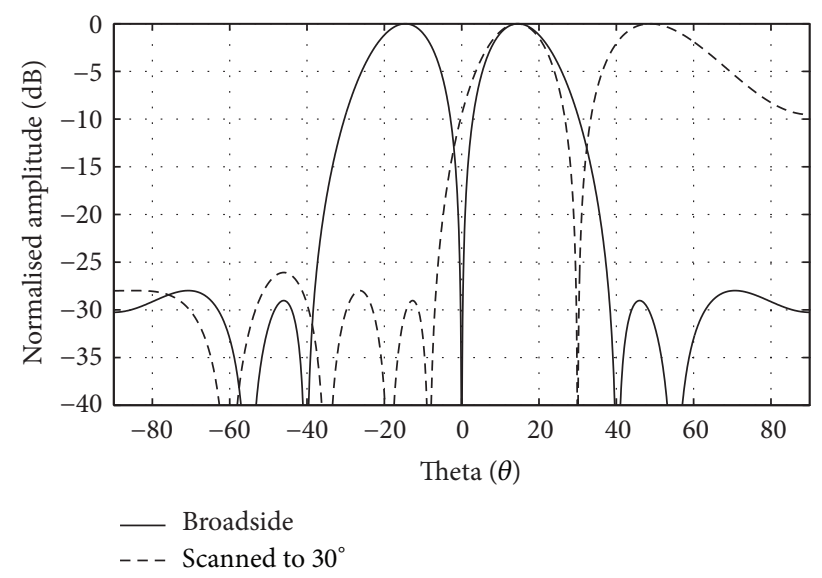

FIGURE 2: Isotropic element array radiation pattern for Bayliss taper with target SLL $=-30 \mathrm{~dB}, d=0.41 \lambda$, and $\overline{\mathbf{n}}=3$, main null scanned to broadside (solid line) and $30^{\circ}$ (dashed line).

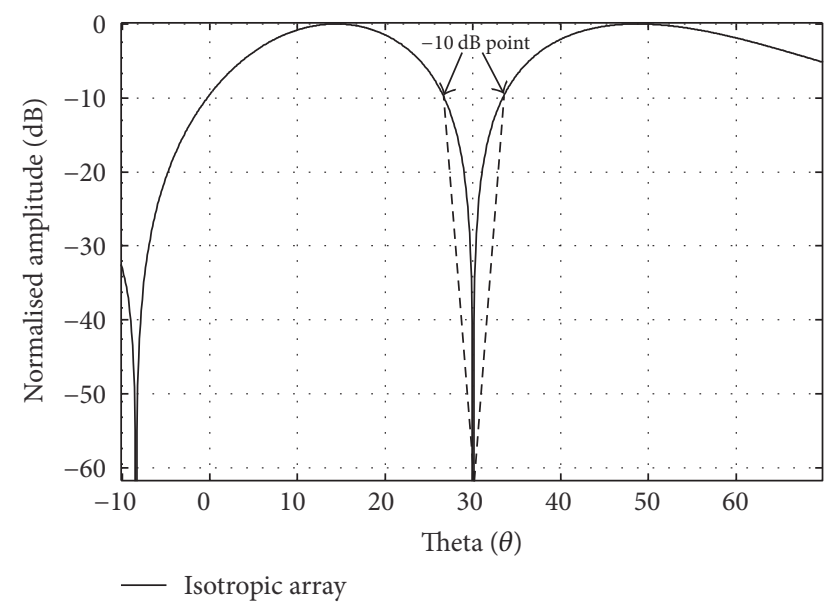

Figure 3: Presentation of slope lines defined to calculate the difference pattern slope.

by the slope of a hypothetical straight line passing through the $-10 \mathrm{~dB}$ point and the main null as highlighted in Figure 2 of isotropic array and expressed as follows:

$$
\kappa=\frac{\operatorname{AF}\left(\theta_{-10 \mathrm{~dB}}\right)-\mathrm{AF}\left(\theta_{o}\right)}{\theta_{o}-\theta_{-10 \mathrm{~dB}}},
$$

where $\operatorname{AF}\left(\theta_{-10 \mathrm{~dB}}\right)$ is the pattern value at $-10 \mathrm{~dB}$ point as indicated in Figure 3, $\mathrm{AF}\left(\theta_{0}\right)$ is main null depth of the difference pattern, and $\theta_{-10 \mathrm{~dB}}$ is the angle value at the indicated $-10 \mathrm{~dB}$ point.

The fitness function used for no SLL restriction is given as follows:

$$
\begin{aligned}
& \psi(\varsigma)=\left[\varsigma_{1}\left(\operatorname{AF}\left(\theta_{1}\right)-\operatorname{AF}\left(\theta_{2}\right)\right)^{2}+\varsigma_{2}\left(\kappa_{\text {iso }}-\kappa_{\text {patt }}\right)^{2}\right. \\
& \left.+\varsigma_{3}\left(\text { NDepth }_{\text {tag }}-\text { NDepth }_{\text {patt }}\right)^{2}\right]^{1 / 2},
\end{aligned}
$$

where $\operatorname{AF}\left(\theta_{1}\right)$ and $\operatorname{AF}\left(\theta_{2}\right)$ are the difference pattern peaks as shown in Figure 4 of BDRA array, $\kappa_{\text {patt }}$ is the minimum of the

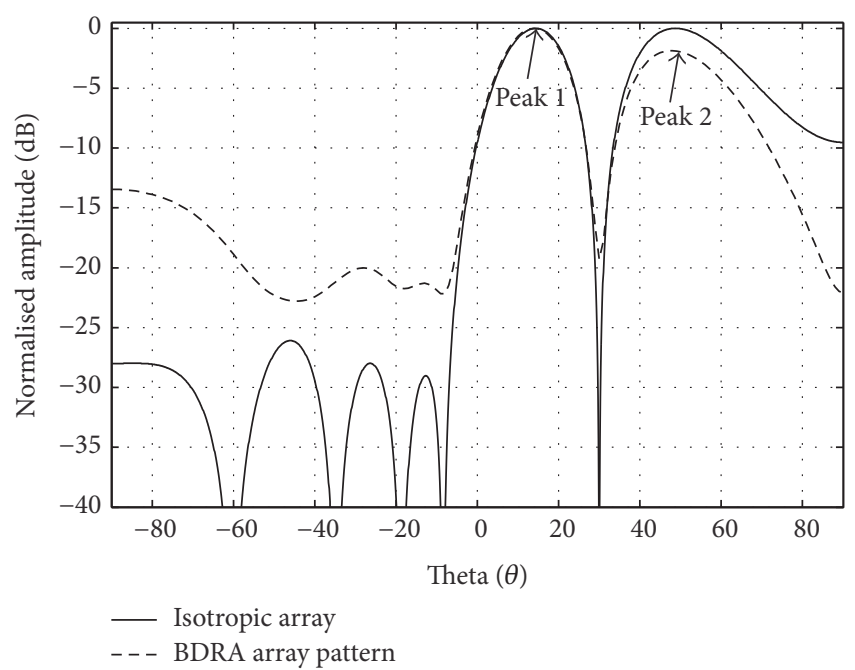

FIgURE 4: Comparison of coupled BDRA array (Equation (4)) and isotropic array patterns for Bayliss distribution with target SLL = $-30 \mathrm{~dB}$ and $\bar{n}=3$ scanned to $30^{\circ}$.

two measured slopes, $\kappa_{\text {iso }}$ is the slope value determined for the isolated BDRA element array, NDepth $_{\text {tag }}$ is the target null depth set to $-60 \mathrm{~dB}$, and $\mathrm{NDepth}_{\text {patt }}$ is the obtained pattern null depth. The slope values are the values of the multipliers that were determined as $\varsigma_{1}=1, \varsigma_{2}=0.08$, and $\varsigma_{3}=0.02$, by following a trial and error procedure. Fitness function used to apply SLL constraint is given in the following:

$$
\begin{aligned}
& \psi(\varsigma)=\left[\varsigma_{1}\left(\operatorname{AF}\left(\theta_{1}\right)-\operatorname{AF}\left(\theta_{2}\right)\right)^{2}+\varsigma_{2}\left(\kappa_{\text {iso }}-\kappa_{\text {patt }}\right)^{2}\right. \\
& +\varsigma_{3}\left(\mathrm{NDepth}_{\text {tag }}-\mathrm{NDepth}_{\text {patt }}\right)^{2} \\
& \left.+\varsigma_{4}\left(\mathrm{SLL}_{\mathrm{des}}-\mathrm{SLL}_{\text {patt }}\right)^{2}\right]^{1 / 2} .
\end{aligned}
$$

The values of the multipliers were determined as $\varsigma_{1}=1, \varsigma_{2}=$ $0.08, \varsigma_{3}=0.02$, and $\varsigma_{4}=0.004$, by following a trial and error procedure.

2.2. Case II. In Case II isotropic element array is considered for monopulse feed simplification with half wavelength separation. It is a well-known fact that a difference pattern can be obtained simply by flipping the phase of the other half of a sum pattern taper. This reduces the complexity of the feeding network but the resultant pattern has high side lobe levels (SLLs). It is a matter of interest here to use a global optimisation technique to find a single excitation set that may produce sum and difference patterns with mere phase flipping within acceptable SLL limits.

$$
\begin{aligned}
& \psi(\varsigma)=\left[\varsigma_{1}\left(\mathrm{SLL}_{\text {des }}^{\text {sum }}-\mathrm{SLL}_{\text {patt }}^{\text {sum }}\right)^{2}\right. \\
& +\varsigma_{2}\left(\mathrm{SLL}_{\mathrm{des}}^{\text {diff }}-\mathrm{SLL}_{\text {patt }}^{\text {diff }}\right)^{2} \\
& \left.+\varsigma_{3}\left(\mathrm{NDepth}_{\text {des }}-\mathrm{NDepth}_{\text {patt }}\right)^{2}\right]^{1 / 2},
\end{aligned}
$$




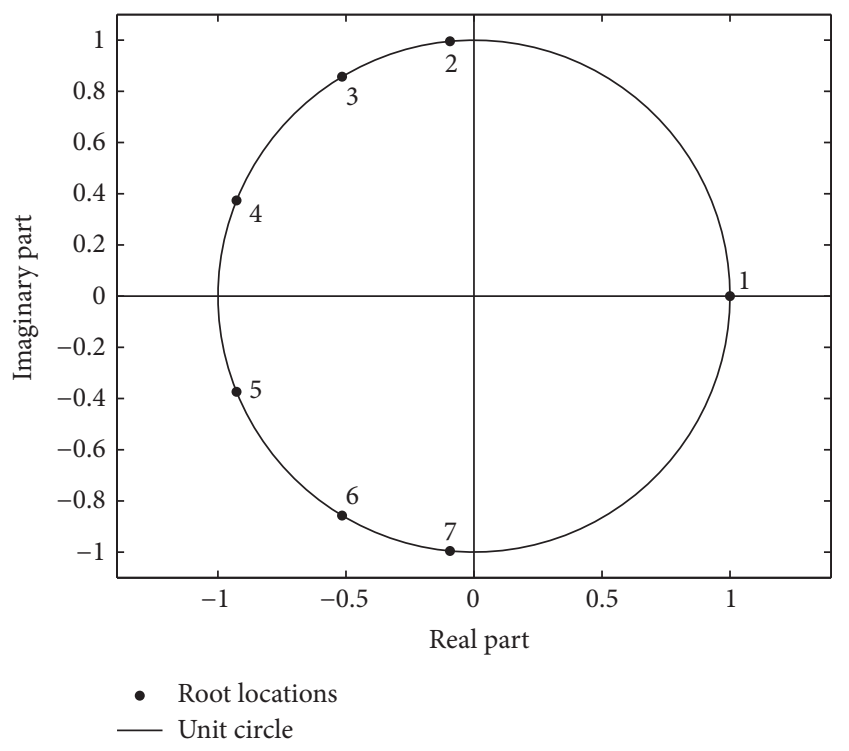

(a)

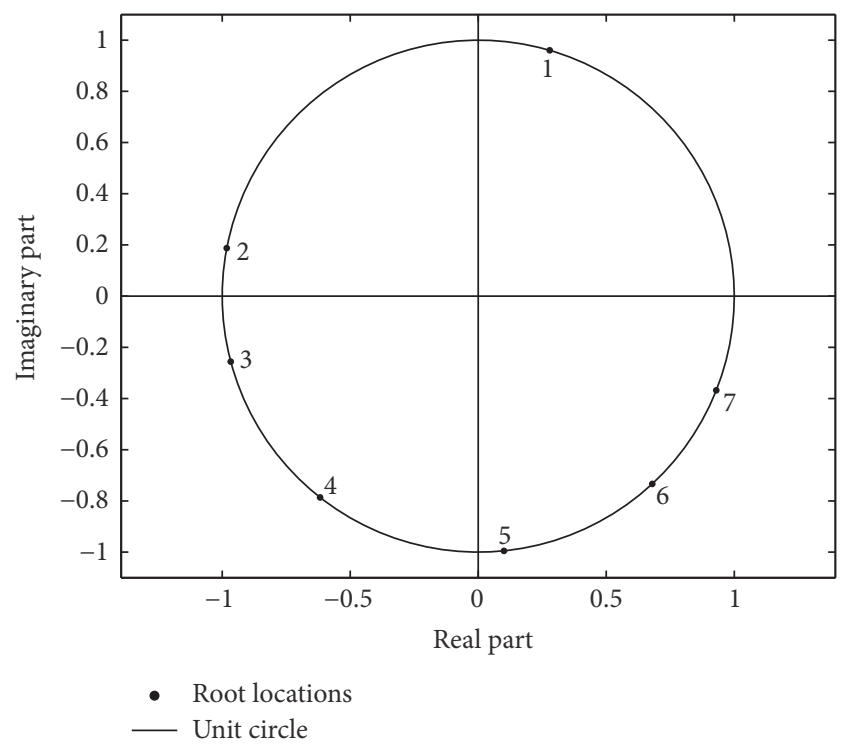

(b)

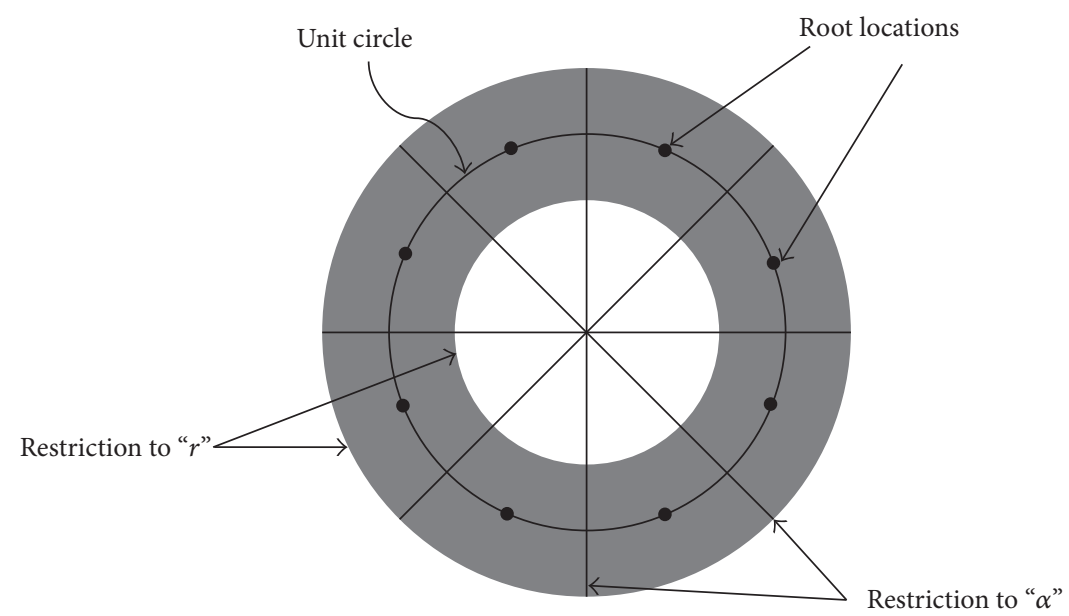

(c)

FIgURE 5: (a) The $z$-space presentation of the Bayliss taper for SLL $=-30 \mathrm{~dB}$. (b) Scanned pattern. (c) General solution space is divided with uniform boundaries.

where $S L L_{\text {des }}^{\text {sum }}$ is desired average SLL for sum pattern which was set to $-20 \mathrm{~dB}$, SLL patt is average SLL obtained for the sum pattern, SLL diff is desired PSLL of the difference pattern which was set to $-15 \mathrm{~dB}$, SLL $\mathrm{diff}$ is PSLL for the obtained difference pattern, NDepth $\mathrm{des}_{\text {is }}$ desired null depth for the main null of the difference pattern which was set to $-40 \mathrm{~dB}$,

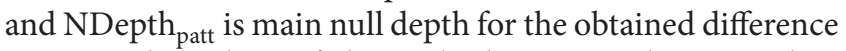
pattern. The values of the multipliers were determined as $\varsigma_{1}=1, \varsigma_{2}=1$, and $\varsigma_{3}=0.8$, by following a trial and error procedure.

The excitation vector corresponding to a proposed optimum root location (values contained by a particle of swarm) was first used to find the sum pattern for which the average SLL was recorded. Then the latter half of the excitation vector was multiplied by -1 (phase flipped by $180^{\circ}$ ) and used to find the difference pattern using the same equation (1).
2.3. Intelligent $z$-Plane Boundary Condition-Particle Swarm Optimiser (IzBC-PSO). IzBC-PSO is an optimisation solver based on the PSO for linear uniformly spaced antenna arrays $[28,33]$. To initialize the IzBC-PSO a null beam width (between the main null and the first null of the difference pattern) is defined. Since we have $N=8$, it means that solution space has to be divided among 14 variables as described in [28] and shown in Figure 5(a). The scanned difference pattern with modified null positions is shown in Figure 5(b) and general root distribution in $z$-plane and boundaries in Figure 5(c). Radiation pattern in $\theta$ space is used and SLLs are compared to determine the bounds on the intermediate root location variable. The root movement is restricted to improve the convergence time as its current location is marked at its lower bound and the upper bound allows roaming within $20 \%$ of $360 /(N-1)$. This restriction may also result in suboptimal convergence which is avoided 
by redefining the variable boundary in steps of $10 \%$ of $360 /(N-1)$. For IzBC-PSO, the swarm size is chosen to be 30 for all cases considered in this work based on investigations reported in [33]. The number of iterations is set to 200 resulting in 6000 fitness function evaluations.

\section{Results and Discussion}

3.1. Case I. Figure 2 shows the isotropic array pattern while main null is scanned to $\theta_{o}=30^{\circ}$. The PSLL is raised to $-26 \mathrm{~dB}$ with average SLL of $-27.8 \mathrm{~dB}$. The difference pattern slope calculated using (5) was found to be $87.8 \mathrm{~dB} / \mathrm{deg}$ with main null as deep as $-300 \mathrm{~dB}$. In the absence of element pattern multiplication effect and the mutual coupling the difference between the twin beam peaks was negligible. All simulations have been performed on a Windows laptop, Intel i5, and 4 GB RAM.

Figure 4 compares the scanned coupled BDRA array and isotropic array radiation patterns for Bayliss taper. For BDRA array radiation pattern PSLL was raised to $-13.4 \mathrm{~dB}$ with the average SLL of $-18 \mathrm{~dB}$ and difference between the twin beam peaks was found to be $-1.8 \mathrm{~dB}$. The null depth and the pattern slope were found to be $-19.3 \mathrm{~dB}$ and $3.1 \mathrm{~dB} / \mathrm{deg}$, respectively. The mutual coupling has significantly affected the pattern characteristics as it is evident from Figure 4.

IzBC-PSO is used to minimize (6) in the presence of mutual coupling and validated by evaluating (4) for each potential solution. The target null depth was set to $-60 \mathrm{~dB}$ and twin beam peaks were required to be at the same level. The difference pattern slope was set to be maximized. The best result obtained out of 10 independent trails of IzBC-PSO is shown in Figure 6. The optimised pattern has null depth of $-60 \mathrm{~dB}$, twin beam peak difference has reduced to $0.02 \mathrm{~dB}$, and slope has improved to $16.7 \mathrm{~dB} / \mathrm{deg}$ as shown in Figure 4 and summarized in Table 1.

To implement SLL constraint (7) is used as the fitness function for IzBC-PSO. The target null depth and SLL were set to $-40 \mathrm{~dB}$ and $-20 \mathrm{~dB}$ for this example. The radiation pattern performance in the presence of mutual coupling has significantly improved after optimisation. The PSLL and average SLL were improved to $-20 \mathrm{~dB}$ and obtained null depth is $-37.3 \mathrm{~dB}$ with the difference pattern slope value of $7.9 \mathrm{~dB} / \mathrm{deg}$. It is evident that optimisation has significantly improved the difference pattern performance compared to the uncompensated coupled BDRA array.

It is shown that by applying the proposed method the effect of pattern multiplication and mutual coupling has been mitigated. The difference in twin beams can be attributed to these factors and will depend on the scan direction. Therefore, for an alternate scan direction a reoptimisation may be required to restore the pattern shape.

3.2. Case II. A Taylor sum pattern with target $-20 \mathrm{~dB}$ SLL and $\bar{n}=6$ was obtained following the conventional procedure $[1,4]$. As the objective was to keep the feed network simple and less expensive only phases of half the element excitations were flipped to give a difference pattern. The resultant difference pattern had a SLL of $-10 \mathrm{~dB}$. The results are shown in Figure 7.

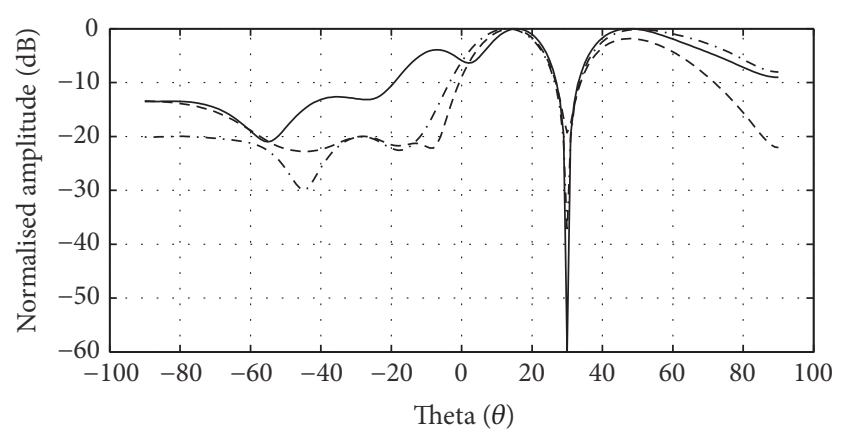

— Optimised radiation pattern using equation (6) (no SLL constraint)

- - - BDRA array pattern

-. - Optimised radiation pattern using equation (7) (SLL constraint)

FIGURE 6: Radiation patterns for BDRA array before and after the optimisation using (6) and (7) for Case I in the presence of mutual coupling.

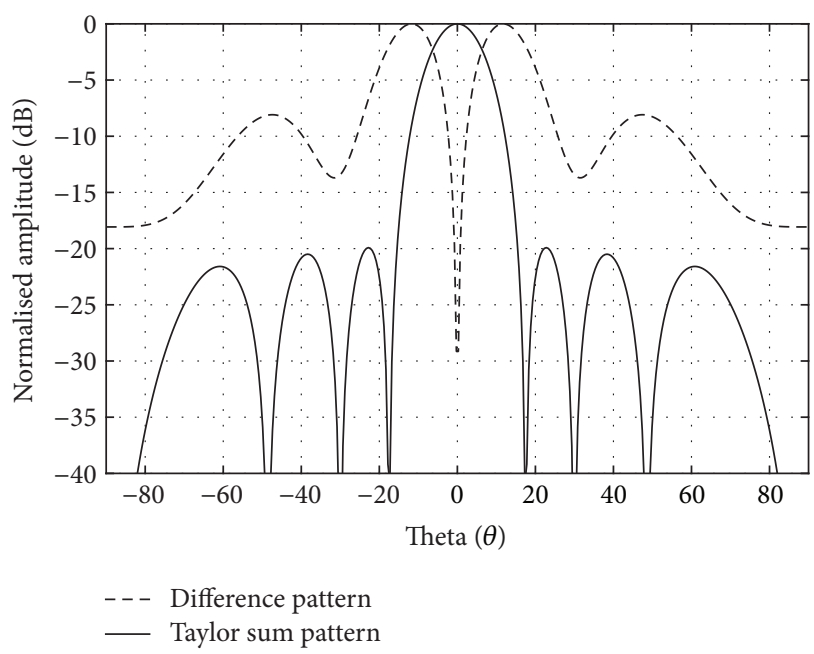

FIgURE 7: Taylor sum pattern for $\overline{\mathbf{n}}=6$ and SLL $=-20 \mathrm{~dB}$, with corresponding difference pattern.

In Figure 8 Bayliss difference pattern for SLL $-15 \mathrm{~dB}$ is shown. Once again to meet the objective, only phases of the half of element excitation were flipped to obtain a sum pattern. The resultant sum pattern shown in Figure 8 has a SLL of $-7.29 \mathrm{~dB}$.

It is clear from the results shown in Figure 7 that the difference pattern obtained from modified Taylor distribution was not acceptable. By the same token, sum pattern obtained by the modified Bayliss excitation was not desirable as evident from Figure 8. A compromise between acceptable difference and sum patterns for small arrays was obtained by using the particle swarm optimiser (PSO). The starting point of the optimisation process was $-20 \mathrm{~dB}$ Taylor sum pattern and then by flipping the sign of the half of the excitation the difference pattern was obtained.

Figure 9 shows that all three objectives were achieved with the sum pattern having the average SLL of $-20.5 \mathrm{~dB}$; the difference pattern has PSLL of $-15 \mathrm{~dB}$ with reference to the difference pattern peak and the null depth of $-40.73 \mathrm{~dB}$. 
TABLE 1: Results summarized from Figures 4 and 6.

\begin{tabular}{|c|c|c|c|c|c|c|}
\hline Array type & $\begin{array}{l}\text { PSLL } \\
(\mathrm{dB})\end{array}$ & $\begin{array}{l}\text { Average } \\
\text { SLL (dB) }\end{array}$ & $\begin{array}{l}\text { Null depth } \\
\quad \text { (dB) }\end{array}$ & $\begin{array}{c}\text { Difference } \\
\text { slope } \\
\text { (dB/deg) }\end{array}$ & $\begin{array}{c}\text { Twin beam } \\
\text { difference } \\
\text { (dB) }\end{array}$ & $\begin{array}{c}\text { Average CPU } \\
\text { time }(\mathrm{sec})\end{array}$ \\
\hline Isotropic element & -26 & -27.8 & -300 & 87.8 & N.A. & - \\
\hline BDRA array & -13.4 & -18 & -19.3 & 3.1 & 1.8 & - \\
\hline IzBC-PSO (Equation (6)) & - & - & -60 & 16.7 & 0.02 & 1.7 \\
\hline IzBC-PSO (Equation (7)) & -20 & -20 & -37.3 & 7.9 & 0.1 & 3.1 \\
\hline
\end{tabular}

TABLE 2: Weight vectors used to determine the patterns in Figure 9.

\begin{tabular}{lccc}
\hline \multicolumn{2}{c}{$\begin{array}{c}\text { Weight vector for sum } \\
\text { pattern }\end{array}$} & \multicolumn{2}{c}{ Weight vector for difference pattern } \\
Amplitude & Phase (deg) & Amplitude & Phase (deg) \\
\hline 0.29 & 0 & 0.29 & 0 \\
0.77 & 0 & 0.77 & 0 \\
1.00 & 0 & 1.00 & 0 \\
0.71 & 0 & 0.71 & 0 \\
0.70 & 0 & 0.70 & 180 \\
0.78 & 0 & 0.78 & 180 \\
0.76 & 0 & 0.76 & 180 \\
0.41 & 0 & 0.41 & 180 \\
\hline
\end{tabular}

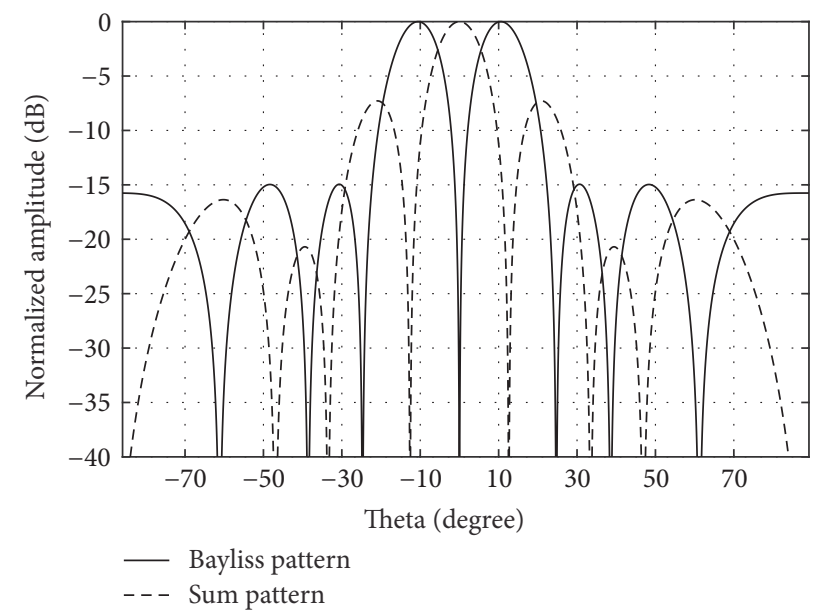

Figure 8: Bayliss difference pattern for $\overline{\mathbf{n}}=3$ and SLL $-15 \mathrm{~dB}$, with corresponding sum pattern.

Optimised weight vectors used to find these patterns are given in Table 2. It is important to note that the magnitude of the excitation taper stays the same for both sum and difference patterns. Only a phase flip of $180^{\circ}$ was applied to achieve the desired difference pattern properties. The improvement in the sum pattern PSLL is $78 \%$ compared to the sum pattern in Figure 8 and array directivity for optimised sum pattern was $8.6 \mathrm{~dB}$. When compared to the Taylor sum pattern directivity $8.9 \mathrm{~dB}$, it is a small cost for having a $50 \%$ feed taper match. The PSLL of the difference pattern shown in Figure 9 have improved by $50 \%$ as compared to the one presented in Figure 7.

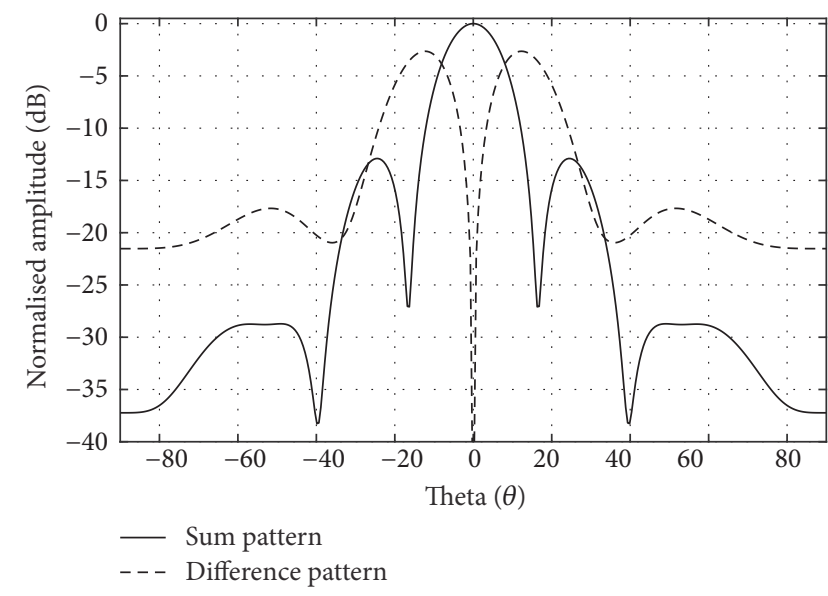

FIGURE 9: Optimised sum and difference patterns.

\section{Conclusion}

It is demonstrated that IzBC-PSO would be a versatile tool to be used as a multiobjective problem optimiser. By applying the proposed solution to the scanning difference pattern array the compromised pattern is improved in terms of average SLL, PSLL, difference pattern slope, and twin beam difference. The objectives are achieved for an array of BDRA elements that includes the mutual coupling and complicates the synthesis problem. It is also shown that the proposed technique has been successfully implemented to simplify the monopulse feed. If the proposed solution is not applied to the situations considered in this paper difference pattern scanning would suffer from pattern degradation and the monopulse feed structure would be more complicated.

\section{Conflicts of Interest}

The authors declare that there are no conflicts of interest regarding the publication of this paper.

\section{References}

[1] T. T. Taylor, "Design of line-source antennas for narrow beamwidth and low side lobes," Transactions of the IRE Professional Group on Antennas and Propagation, vol. 3, no. 1, pp. 16-28, 1955.

[2] D. A. McNamara, "Direct synthesis of optimum difference patterns for discrete linear arrays using Zolotarev distributions," IEE Microwaves, Antennas and Propagation Proceedings, vol. 140, no. 6, p. 495, 1993. 
[3] E. T. Bayliss, "Design of monopulse antenna difference patterns with low sidelobes," Bell System Technical Journal, vol. 47, no. 5, pp. 623-650, 1968.

[4] R. S. Elliott, Antenna Theory and Design, IEEE Press, John Wiley and Sons, New Jersey, NJ, USA, 2003.

[5] C. L. Dolph, "A current distribution for broadside arrays which optimizes the relationship between beam width and side-lobe level," Proceedings of the IRE, vol. 34, no. 6, pp. 335-348, 1946.

[6] D. A. McNamara, "Performance of Zolotarev and modifiedZolotarev difference pattern array distributions," IEE Proceedings: Microwaves, Antennas and Propagation, vol. 141, no. 1, pp. 37-44, 1994.

[7] S. A. Hosseini and Z. Atlasbaf, "Optimisation of side lobe level and fixing quasi-nulls in both of the sum and difference patterns by using continuous ant colony optimisation method," Progress in Electromagnetics Research, vol. 79, pp. 321-337, 2008.

[8] P. Rocca, L. Manica, and A. Massa, "Synthesis of monopulse antennas through iterative contiguous partition method," Electronics Letters, vol. 43, no. 16, pp. 854-856, 2007.

[9] M. D'Urso and T. Isernia, "Solving some array synthesis problems by means of an effective hybrid approach," IEEE Transactions on Antennas and Propagation, vol. 55, no. 3, pp. 750-759, 2007.

[10] M. Álvarez-Folgueiras, J. A. Rodríguez-González, and F. AresPena, "Synthesising Taylor and Bayliss linear distributions with common aperture tail," Electronics Letters, vol. 45, no. 1, pp. 1819, 2009.

[11] O. M. Bucci, M. D’Urso, and T. Isernia, “Optimal synthesis of difference patterns subject to arbitrary sidelobe bounds by using arbitrary array antennas," EEE Proceedings Microwaves, Antennas and Propagation, vol. 152, no. 3, pp. 129-137, 2005.

[12] F. Ares, S. Rengarajan, A. Vieiro, and E. Moreno, "Optimisation of aperture distributions for difference patterns," in Proceedings of the IEEE Antennas and Propagation Society International Symposium, vol. 4, pp. 1826-1829, Newport Beach, Calif, USA.

[13] P. López, J. A. Rodríguez, F. Ares, and E. Moreno, "Subarray weighting for the difference patterns of monopulse antennas: joint optimization of subarray configurations and weights," IEEE Transactions on Antennas and Propagation, vol. 49, no. 11, pp. 1606-1608, 2001.

[14] A. A. Khan and A. K. Brown, "Difference pattern synthesis using a particle swarm optimiser," in Proceedings of the Loughborough Antennas and Propagation Conference, pp. 329-332, 2009.

[15] D. A. McNamara, "Synthesis of sub-arrayed monopulse linear arrays through matching of independently optimum sum and difference excitations," IEE Proceedings H: Microwaves, Antennas and Propagation, vol. 135, no. 5, pp. 293-296, 1988.

[16] P. Rocca, L. Manica, and A. Massa, "An improved excitation matching method based on an ant colony optimization for suboptimal-free clustering in sum-difference compromise synthesis," IEEE Transactions on Antennas and Propagation, vol. 57, no. 8, pp. 2297-2306, 2009.

[17] A. Massa, M. Pastorino, and A. Randazzo, "Optimization of the directivity of a monopulse antenna with a subarray weighting by a hybrid differential evolution method," IEEE Antennas and Wireless Propagation Letters, vol. 5, no. 1, pp. 155-158, 2006.

[18] M. D’Urso, T. Isernia, and E. F. Meliado, "An effective hybrid approach for the optimal synthesis of monopulse antennas," IEEE Transactions on Antennas and Propagation, vol. 55, no. 4, pp. 1059-1066, 2007.
[19] L. Manica, P. Rocca, A. Martini, and A. Massa, "An innovative approach based on a tree-searching algorithm for the optimal matching of independently optimum sum and difference excitations," IEEE Transactions on Antennas and Propagation, vol. 56, no. 1, pp. 58-66, 2008.

[20] L. Manica, P. Rocca, M. Pastorino, and A. Massa, "Boresight slope optimization of subarrayed linear arrays through the contiguous partition method," IEEE Antennas and Wireless Propagation Letters, vol. 8, pp. 253-257, 2008.

[21] P. Rocca, L. Manica, R. Azaro, and A. Massa, "A hybrid approach to the synthesis of subarrayed monopulse linear arrays," IEEE Transactions on Antennas and Propagation, vol. 57, no. 3, pp. 652-663, 2009.

[22] Y. Chen, S. Yang, and Z. Nie, "The application of a modified differential evolution strategy to some array pattern synthesis problems," IEEE Transactions on Antennas and Propagation, vol. 56, no. 7, pp. 1919-1927, 2008.

[23] L. Manica, P. Rocca, and A. Massa, "Excitation matching procedure for sub-arrayed monopulse arrays with maximum directivity," IET Radar, Sonar and Navigation, vol. 3, no. 1, pp. 280-283, 2009.

[24] T.-S. Lee and T.-K. Tseng, "Subarray-synthesized low sidelobe sum and difference patterns with partial common weights," IEEE Transactions on Antennas and Propagation, vol. 41, no. 6, pp. 791-800, 1993.

[25] S. Caorsi, A. Massa, M. Pastorino, and A. Randazzo, "Optimization of the difference patterns for monopulse antennas by a hybrid real/integer-coded differential evolution method," IEEE Transactions on Antennas and Propagation, vol. 53, no. 1, pp. 372-376, 2005.

[26] J. R. Mohammed, "Synthesizing sum and difference patterns with low complexity feeding network by sharing element excitations," International Journal of Antennas and Propagation, vol. 2017, Article ID 2563901, 7 pages, 2017.

[27] Y. Liu, Y.-C. Jiao, Y.-M. Zhang, and Y.-Y. Tan, "Synthesis of phase-only reconfigurable linear arrays using multiobjective invasive weed optimization based on decomposition," International Journal of Antennas and Propagation, vol. 2014, Article ID 630529, 11 pages, 2014.

[28] A. A. Khan and A. K. Brown, "Intelligent Z-plane boundary condition particle swarm optimiser (IzBC-PSO) for small array pattern synthesis," IET Microwave Antennas and Propagation, vol. 6, no. 14, pp. 1598-1607, 2012.

[29] L. Z. Thamae and Z. Wu, "Broadband bowtie dielectric resonator antenna," IEEE Transactions on Antennas and Propagation, vol. 58, no. 11, pp. 3707-3710, 2010.

[30] P.-S. Kildal, A. Vosoogh, and S. MacI, "Fundamental directivity limitations of dense array antennas: a numerical study using hannan's embedded element efficiency," IEEE Antennas and Wireless Propagation Letters, vol. 15, pp. 766-769, 2016.

[31] B. Sun, C. Liu, Y. Liu, X. Wu, Y. Li, and X. Wang, "Conformal array pattern synthesis and activated elements selection strategy based on PSOGSA algorithm," International Journal of Antennas and Propagation, vol. 2015, Article ID 858357, 7 pages, 2015.

[32] A. T. Villeneuve, "Taylor patterns for discrete arrays," IEEE Transactions on Antennas and Propagation, vol. 32, no. 10, pp. 1089-1093, 1984.

[33] I. C. Trelea, “The particle swarm optimization algorithm: convergence analysis and parameter selection," Information Processing Letters, vol. 85, no. 6, pp. 317-325, 2003. 


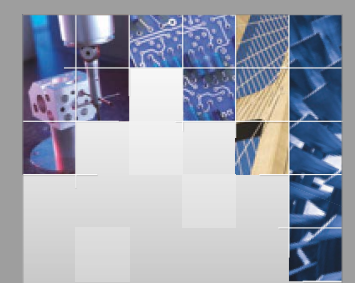

\section{Enfincering}
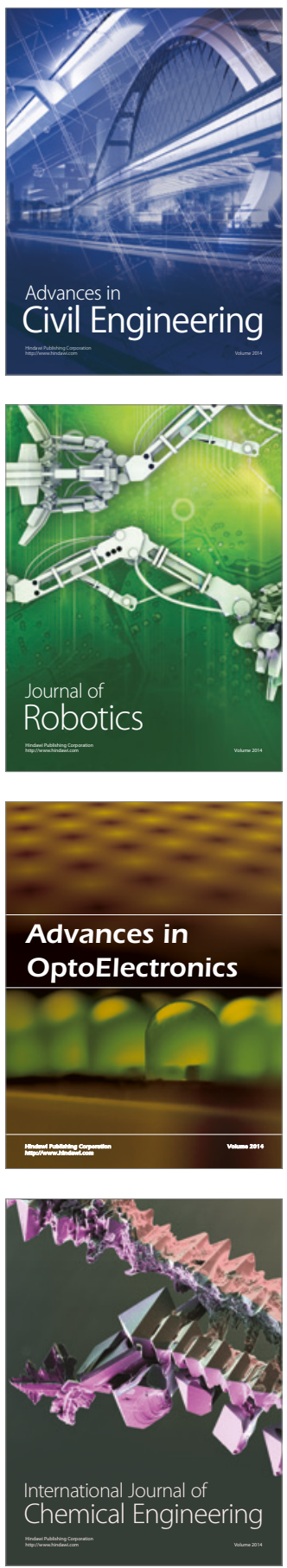

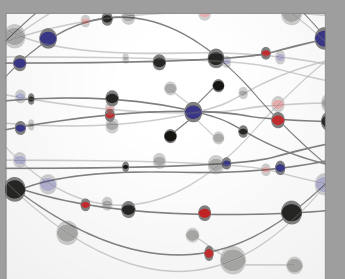

The Scientific World Journal

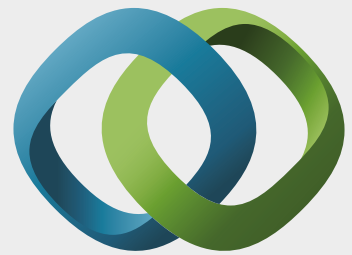

\section{Hindawi}

Submit your manuscripts at

https://www.hindawi.com
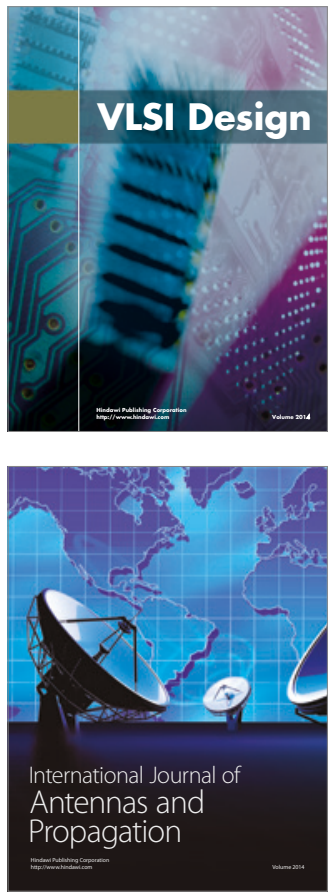

\section{Rotating}

Machinery
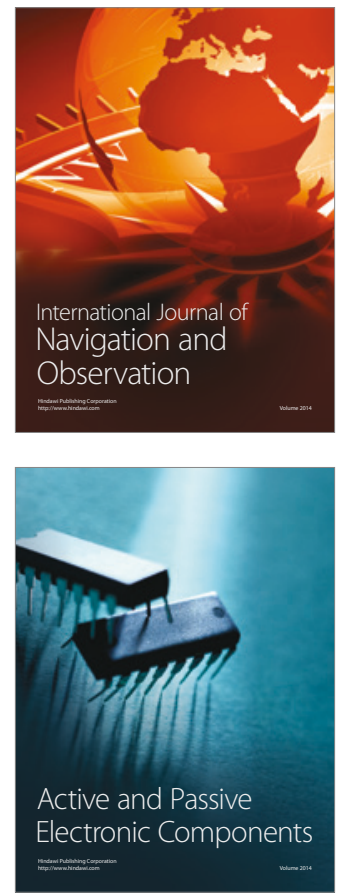
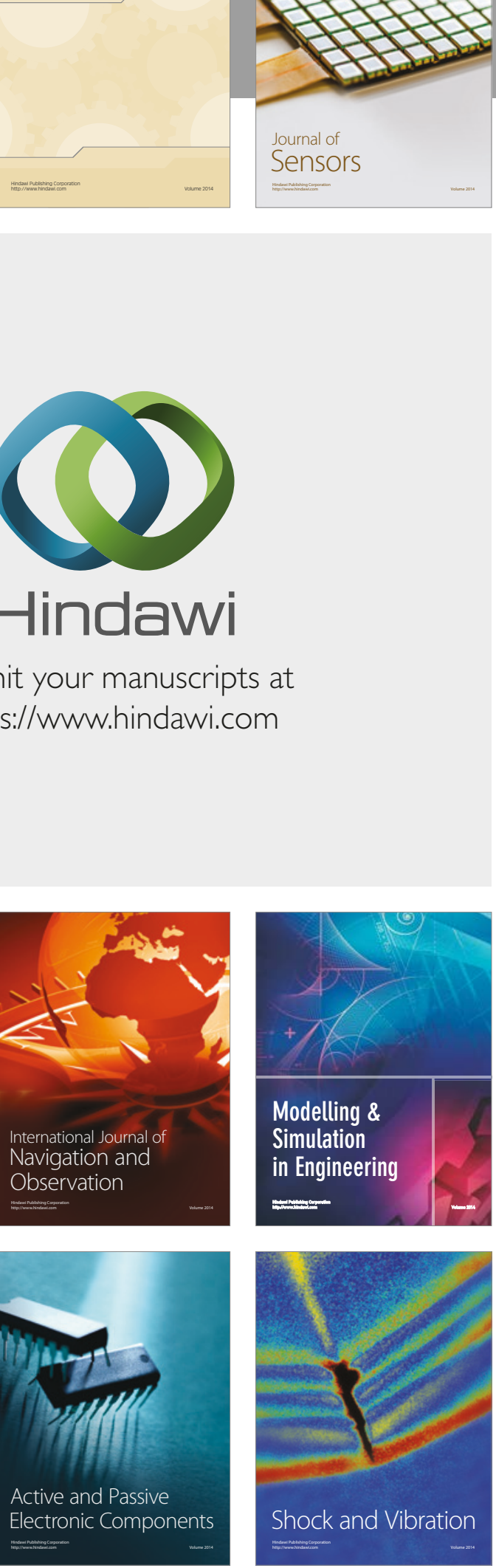
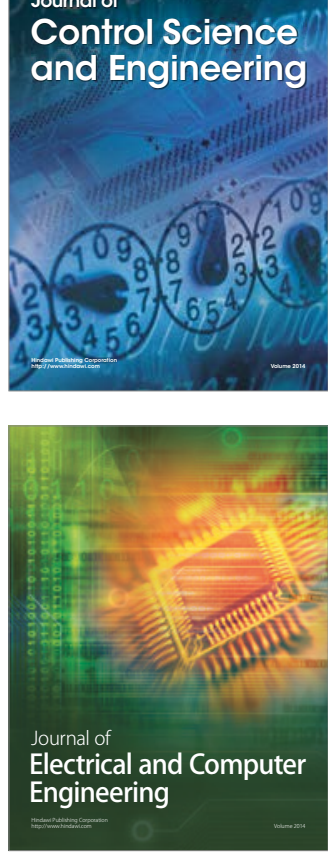

Distributed

Journal of

Control Science

and Engineering
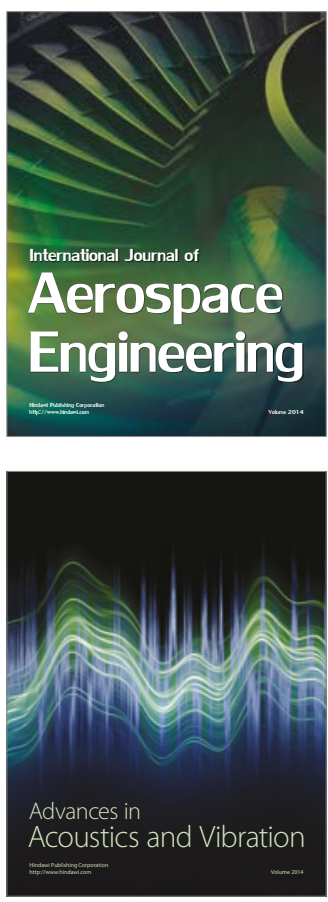

Sensor Networks 